\title{
Making mirrors for giant telescopes
}

\author{
H. Martin
}

H. M. Martin, "Making mirrors for giant telescopes," Proc. SPIE 11116, Astronomical Optics: Design, Manufacture, and Test of Space and Ground Systems II, 111160J (9 September 2019); doi: 10.1117/12.2534601

SPIE Event: SPIE Optical Engineering + Applications, 2019, San Diego, California, SPIE. United States 


\title{
Making mirrors for giant telescopes
}

\author{
H. M. Martin \\ Steward Observatory and College of Optical Sciences, University of Arizona, Tucson, AZ 85721, \\ USA
}

\begin{abstract}
Advances in our understanding of the Universe depend on improvements in sensitivity and angular resolution that can come only with larger telescopes. Telescope diameters increased by almost an order of magnitude in the last century, but that growth has been sporadic, limited mainly by the ability to make bigger mirrors that hold their shape against the dynamic effects of gravity, wind and temperature. Three major advances in mirror technology occurred in the 1980s, including the lightweight honeycomb mirrors made at the Richard F. Caris Mirror Lab at the University of Arizona. In this informal paper, I will describe these technologies and show how they enabled the current generation of 8- to $12-\mathrm{m}$ telescopes and how they are now being used to build telescopes of 25 to $39 \mathrm{~m}$.
\end{abstract}

Keywords: telescopes, ELTs, Giant Magellan Telescope, optical fabrication, optical testing

\section{100 YEARS AGO}

Edwin Hubble arrived at Mt. Wilson Observatory near Los Angeles in 1919, to start observing with the new 100 inch Hooker telescope. The 100 inch telescope had been commissioned in 1917, becoming the world's largest telescope and the first to surpass Lord Rosse's 72 inch telescope from 1845. More significantly, the 100 inch telescope more than doubled the collecting area of the Mt. Wilson 60 inch telescope, which was much more advanced than Rosse's and the most powerful telescope ever built when it was completed in 1908.
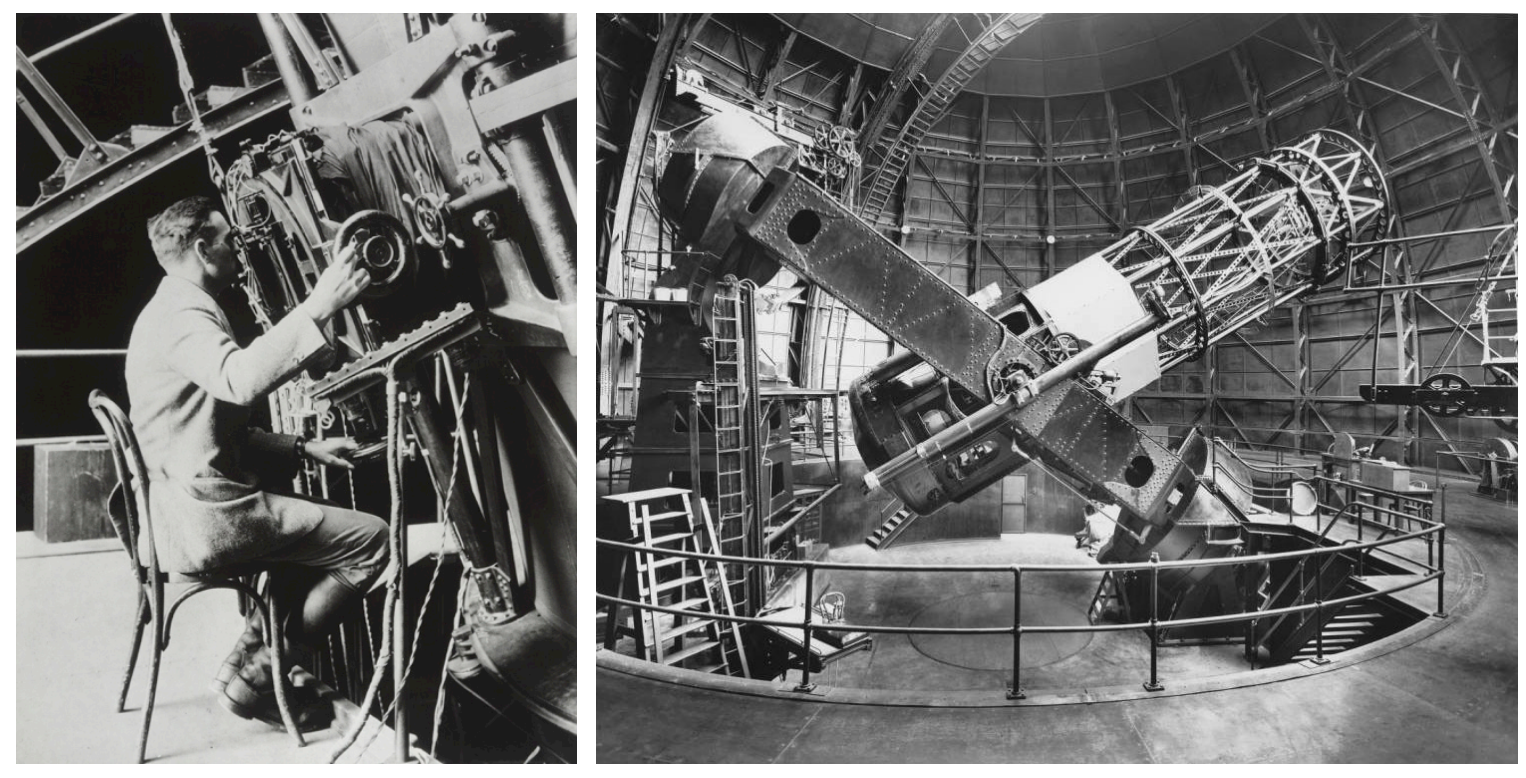

Figure 1. Left: Edwin Hubble observing with the 100 inch Hooker telescope at Mount Wilson Observatory in 1924. Edwin Hubble Papers/Courtesy of Huntington Library, San Marino, CA. Right: The 100 inch telescope. Courtesy of the Observatories of the Carnegie Institution for Science Collection at the Huntington Library, San Marino, Calif.

One of Hubble's first projects was to photograph stars in the spiral nebulae, as they were called, generally thought to be clouds of gas and stars in the Milky Way. There was evidence going back to the mid-19 $9^{\text {th }}$ century that spiral nebulae 
were actually distant galaxies, and a debate was underway, but in 1919 many, probably most astronomers thought the Milky Way was the entire Universe.

Hubble's photographs with the 100 inch telescope resolved individual stars more distinctly than any previous observations. In 1923 he identified a Cepheid variable star in the Andromeda nebula, similar to thousands of known Cepheid variables but much fainter. Henrietta Swan Leavitt had recently discovered a strong relation between the period of variation in brightness of this class of stars and their luminosity, which Hubble used to determine the distance and show that this star and therefore the Andromeda nebula was about 900,000 light-years away, roughly 10 times the diameter of the Milky Way. (Later re-calibration of the Cepheid relation would push Andromeda out to over 2 million light-years.) Within a few years Hubble identified galaxies 6 times farther than Andromeda. By 1931, using other distance indicators in addition to variable stars, Hubble had expanded the known Universe by a factor of 1300 in distance or 2 billion in volume. ${ }^{[1]}$
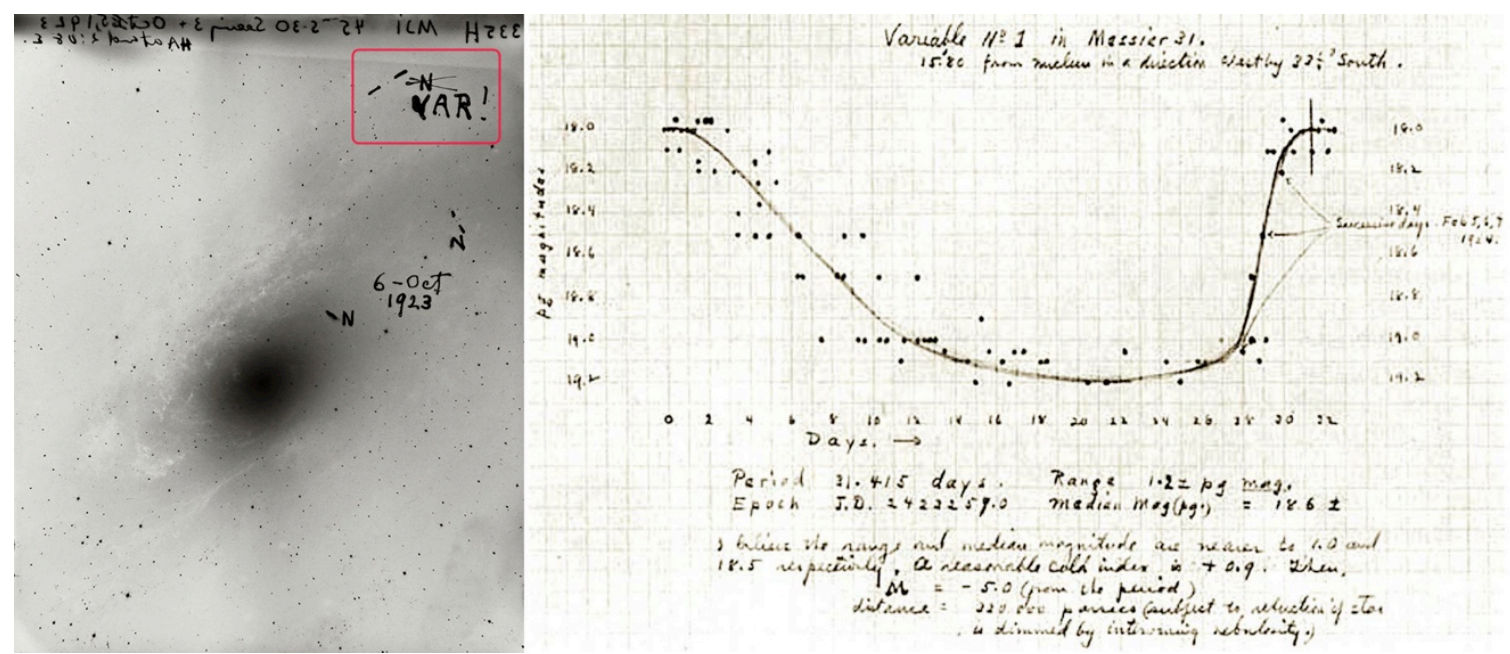

Figure 2. Left: part of Hubble's photographic plate indicating a Cepheid variable star at the edge of the Andromeda Nebula (initially marked $\mathrm{N}$ for nova, then corrected to VAR! as Hubble realized he had found a distance indicator). Right: Light curve showing the 31.4 day period of variation and Hubble's early estimate of distance, 220,000 parsecs or 720,000 light-yr. Courtesy of Carnegie Observatories.

The debate about the nature of spiral nebulae was over. Hubble's observations with the new, world's largest telescope had definitively established that the Milky Way is one of thousands (later, billions) of galaxies in a Universe that's orders of magnitude larger than previously thought.

And this isn't even what Hubble is most famous for.

By 1929 Hubble could combine his distances to galaxies with redshifts measured by Vesto Slipher at Lowell Observatory and Milton Humason at Mt. Wilson, to show (a) all galaxies at distances more than about 10 million lightyears were moving away from us, and (b) there's a linear relation between distance and recession speed. ${ }^{[1]}$ The only reasonable explanation was that the Universe is expanding. Not only was the Universe dynamic on the largest possible scales, but one could play the expansion back in time to a birth day, the Big Bang. Hubble's data put it 2 billion years ago, but later revisions of the distance scale give us the present age of about 14 billion years.

All of this happened within a decade of Hubble's first observations with the 100 inch telescope, and within a dozen years of its commissioning. This is what can happen when you build a telescope almost twice the size of previous telescopes (and when it's used by someone with Hubble's understanding of the important astrophysical problems it can address). The 100 inch telescope brought one significant improvement over the 60 inch: collecting area. This was a time when detectors (photographic plates) were stuck at $1 \%$ efficiency, when angular resolution was limited not by diffraction but by the atmosphere (seeing) as well as the performance of the mount and the method of manual fine guiding. The 100 inch telescope was little or no better than the 60 inch in these respects. ${ }^{1}$ But the improvement in sensitivity was enough

\footnotetext{
${ }^{1}$ My interpretation of the discussion in [2].
} 
to revolutionize our understanding of the Universe like never before, at least not since Copernicus moved earth away from the center of the Universe.

Jumping back to 2019, construction is underway for a new generation of telescopes that are roughly twice the size of existing telescopes and bring at least the same improvement in sensitivity that Hubble was able to exploit. What's more, with adaptive optics becoming routine at infrared wavelengths and gradually moving to visible wavelengths, the new telescopes will also have much better angular resolution. They are among the first telescopes to have adaptive optics built into the basic design and to anticipate diffraction-limited performance in many observing modes. The combined improvements in sensitivity and resolution are unprecedented. Dramatic discoveries are unpredictable, but we shouldn't be surprised to see some drama in the next decade.

\section{MIRRORS}

All else being equal, bigger telescopes produce more knowledge and at a higher rate. Astronomers have always worked toward building and using the largest telescopes possible. But what determines how big a telescope can be built? Why did the 100 inch telescope become available in 1917? The 200 inch in 1949? The first Keck $10 \mathrm{~m}$ telescope in 1993? The next generation of 25- to 39-m telescopes sometime in the 2020s? For more than a century, the answer has been mirrors.

The last time the primary mirror was not, at least arguably, the biggest technical challenge in the largest telescope built to date was the 60 inch telescope at Mt. Wilson. George Hale's father had bought a 60 inch disk in 1896 when Hale was setting up Yerkes Observatory near Chicago. The St. Gobain glassworks was comfortably able to pour and anneal the $800 \mathrm{~kg}$ blank. Hale had hired George Ritchey as optician and telescope builder at Yerkes. The two moved to Mt. Wilson after Hale's Solar Observatory was established there, and Ritchey figured the 60 inch mirror with no great difficulty. In doing so he established methods that became the standard for more than 50 years, including starting with an edgematching sphere so that no further figuring would have to be done at the always difficult edge, and systematic knife-edge testing to measure the radius of curvature as a function of radial position on the mirror. ${ }^{[2]}$

Anticipating a larger telescope before the 60 inch was completed, Hale ordered a 100 inch disk from St. Gobain in 1906. This proved to be just beyond their ability to make a high-quality mirror blank, and no other vendor had more capability. The large volume of glass required pouring from 3 separate pots and the result was large concentrations of bubbles at the interfaces. The disk was judged unusable and the glassworks made 3 more attempts over the next 2 years, but none of them succeeded. In 1910 Hale directed Ritchey to try to figure the original blank, which he did over the next 5 years, in the end making an excellent mirror whose bubbles had little or no influence. The first images obtained with the telescope were poor, but this turned out to be due to avoidable heating of the mirror during the day, and before morning the telescope was delivering excellent images, as it did for Hubble's ground-breaking work over the next 15 years. ${ }^{2}$

Soon after the commissioning of the 100 inch telescope, Hale and colleagues were working on plans for a larger telescope. By 1928 Hale had decided on 200 inches. Design and fabrication of a new type of mirror was the greatest challenge for the project. Even apart from the difficulty of making the 100 inch mirror blank, its thermal behavior reinforced the understanding that this was the limit for a solid mirror of plate glass with its high expansion coefficient, 8 parts per million per K. Since 1904 Hale and Ritchey had been working with Elihu Thomson of General Electric in a low-level effort to form mirror blanks out of fused quartz with its much lower expansion, $0.6 \mathrm{ppm} / \mathrm{K}$. The Rockefeller Foundation approved funding for the telescope in 1928 and the serious development of fused quartz started then. Thomson experienced problem after problem and had managed to make only a 24 inch blank by 1931, when Hale pulled the plug.

Hale already had a backup plan for a lightweighted mirror out of Pyrex with an intermediate thermal expansion of $3 \mathrm{ppm} / \mathrm{K}$. Corning had been making Pyrex for cookware since 1915 and had made several solid mirror blanks. The 200 inch mirror would have a facesheet 5 inches thick and an open back with pockets to give it about half the mass of a solid mirror. Corning started work in 1931, building a high-temperature gas furnace to melt the glass and an electric furnace, containing the mold, with good temperature control for the 8 months of cooling and annealing. They made the first attempt in 1934, but it failed when some of the mold cores for lightweighting broke free. After a quick modification of the mold, a second pouring was made in December 1934, leading to the successful 200 inch mirror blank.

\footnotetext{
${ }^{2}$ Much of the material in this section comes from Pauper and Prince ${ }^{[3]}$, Donald Osterbrock's fascinating account of Hale and Ritchey, two of the great minds in the history of telescopes, who managed to work together long enough to build the 60 inch and 100 inch telescopes before conflicts between them led to Ritchey's banishment from US astronomy.
} 
Ritchey had had a major falling-out with Hale and was not involved in figuring the 200 inch mirror, but the methods he developed for the 60 inch and 100 inch mirrors were used. The 200 inch mirror's focal ratio is 3.3 (vs 5 for the 60 inch and 100 inch), which allowed a smaller and less expensive dome, shorter exposures with photographic plates, and a wider field for extended objects like nebulae. This made the 200 inch mirror 7 times more aspheric than the 100 inch and by far the most aspheric mirror made to date. The figuring extended from 1936 to 1947-partly because of the large volume of glass to be removed from the edge-matching sphere and partly because of the war-and some refiguring was done in 1949 after initial tests in the telescope. In the end, for the second time, the world's largest mirror had been made successfully after the initial attempts made the result doubtful.

The 200 inch telescope remained the world's most powerful telescope for 44 years, an extraordinary period for a single instrument to hold that distinction in a vibrant field of science. "Most powerful" is arguable in the second half of that period - with dramatic improvements in instruments at all telescopes, better testing technology, and evolving performance standards - but in any case the 200 inch telescope remained the largest telescope with good image quality. The reason was that no one could figure out how to make a larger mirror that would hold its shape to the accuracy required. The telescope should add very little blurring to the best images delivered by the atmosphere-around 0.3 arcsecond - and the implied surface accuracy is around $100 \mathrm{~nm} \mathrm{rms}$. The mirror has to hold this shape accuracy against changing gravity loads as it sweeps across the sky, wind up to around $10 \mathrm{~m} / \mathrm{s}$, and changing air temperature in the open dome. Thermal issues include distortion of the mirror due to its temperature gradients and wavefront distortion by turbulent air in contact with a warm mirror (mirror seeing). A solid 6 meter mirror, in the Soviet Union's BTA-6 that was commissioned in 1975, failed to deliver good images primarily because of its huge thermal inertia, ${ }^{[2]}$ and hasn't been as productive as the 200 inch telescope and a number of smaller telescopes built in the 1970s and 80 s. Telescopes wouldn't break through the $5 \mathrm{~m}$ barrier until the first Keck telescope, with a $10 \mathrm{~m}$ segmented primary mirror, came on-line in 1993. (The Hubble Space Telescope was repaired, to achieve superb image quality, in the same year.)

\section{NEW TECHNOLOGIES FOR BIGGER MIRRORS}

The two Keck telescopes represent one of three new mirror technologies that emerged in the 1980s. These technologies led to the current generation of 8- to 12-m telescopes and continue to be enabling technologies for the next generation of 25- to 39-m telescopes. Jerry Nelson and colleagues at the University of California and Caltech had the bold idea that they could make a $10 \mathrm{~m}$ primary mirror out of 36 segments, each $1.8 \mathrm{~m}$ in diameter. The segments would form a continuous parabolic surface, held to optical tolerances by positioning actuators with feedback from wavefront sensors and electrical displacement sensors. Each segment was small enough $(75 \mathrm{~mm}$ thick) that it would hardly distort under its own weight. The segments were made of Schott's Zerodur glass ceramic with essentially zero thermal expansion. ${ }^{[4]}$

This concept has worked extremely well in the Keck telescopes, was used for three more $10 \mathrm{~m}$ telescopes, and is the core technology for two of the three extremely large telescopes (ELTs) being built today: the Thirty Meter Telescope $\left(\right.$ TMT $^{[5]}$ with $4921.4 \mathrm{~m}$ segments and the European Southern Observatory's 39 m ELT $^{[6]}$ with $7981.4 \mathrm{~m}$ segments.
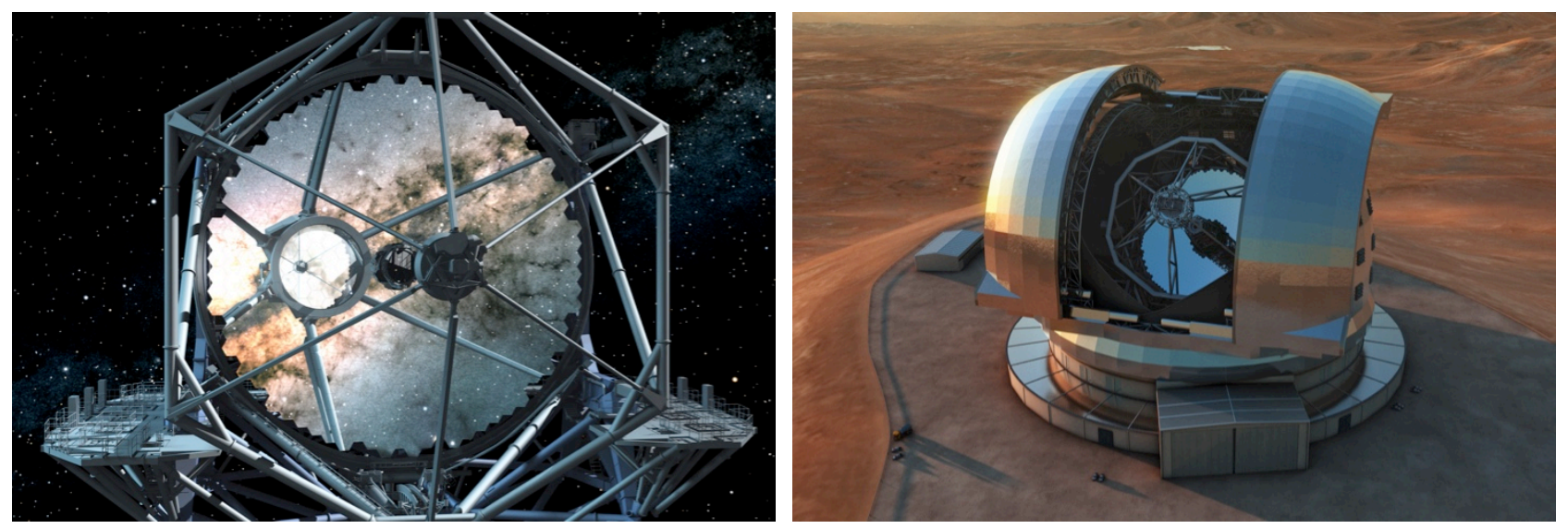

Figure 3. Left: Drawing of the TMT telescope assembly. Courtesy of TMT International Observatory. Right: Drawing of the ESO 39 m ELT. Courtesy of ESO/L. Calçada. 
At around the same time Nelson was developing segmented mirrors, Ray Wilson and colleagues at the European Southern Observatory (ESO) decided they could give up the stiffness of a thick mirror and instead control the shape of a large, relatively flexible mirror, again with actuators and wavefront sensors. In this case the actuators apply controlled force rather than hold controlled position; about 150 actuators are used to control the shape of an $8 \mathrm{~m}$ mirror that's 175 $200 \mathrm{~mm}$ thick. This method of maintaining an accurate mirror surface with force actuators and wavefront sensing is called active optics. It is now built into all $8 \mathrm{~m}$ class telescopes and is an essential element of all three next-generation ELTs. ${ }^{[7],[8]}$

Seven $8 \mathrm{~m}$ telescopes, including ESO's 4-telescope VLT, have similar thin meniscus mirrors made of Zerodur or Corning's zero-expansion ULE. A key aspect of the concept is that Schott and Corning developed the ability to make these thin $8 \mathrm{~m}$ mirrors, not without some struggles in the process. Similar mirrors are used as the $3.4 \mathrm{~m}$ secondary mirror of the Large Synoptic Survey Telescope (LSST) and the $4 \mathrm{~m}$ secondary and tertiary mirrors of ESO's ELT.

Also around the same time, Roger Angel at the University of Arizona developed a way to maintain the stiffness of traditional mirrors but with a small fraction of the weight, extending the 200 inch design to $8 \mathrm{~m}$ and with more extreme lightweighting. The key technology is spin-casting with a honeycomb mold that removes $80 \%$ of the volume of a solid mirror. Low-expansion borosilicate glass (similar to Pyrex) is the best material that can be cast in this complex structure; it's not possible with the zero-expansion materials, which would have to be machined to achieve this kind of lightweighting. In addition to their high stiffness:weight ratio, honeycomb mirrors have rapid thermal response because of their thin glass sections. With simple ventilation, they stay nearly isothermal and follow the changing ambient temperature to minimize mirror seeing. ${ }^{[9]}$

Angel's honeycomb mirrors were the enabling technology behind the MMT and Magellan $6.5 \mathrm{~m}$ telescopes and the Large Binocular Telescope (LBT) with its twin $8.4 \mathrm{~m}$ primary mirrors. The same mirror design was used for LSST's combined $8.4 \mathrm{~m}$ primary and $5.1 \mathrm{~m}$ tertiary mirrors. And these honeycomb mirror are the heart of the Giant Magellan Telescope (GMT), whose $25 \mathrm{~m}$ primary mirror consists of seven $8.4 \mathrm{~m}$ honeycomb segments. ${ }^{[10]}$

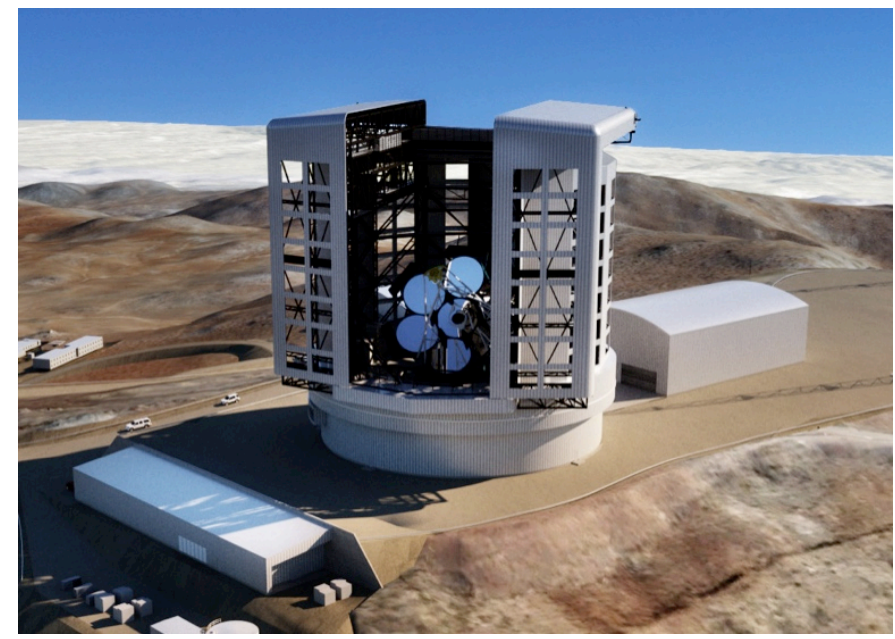

Figure 4. Drawing of the GMT at Las Campanas Observatory in Chile. Courtesy of Giant Magellan Telescope - GMTO Corporation.

\section{DEVELOPMENT OF HONEYCOMB MIRRORS}

Angel and John Hill started experimenting with casting borosilicate in complex molds around 1980. They worked in a fast and furious mode for 2-3 years, going through a wide variety of schemes for forming the honeycomb mirror and holding the mold pieces in places. Figure 5 reproduces several figures from a single 1982 SPIE paper ${ }^{[11]}$ showing three methods of casting the mirror right-side up, and one upside down that was briefly the preferred option as indicated by the sketch of a future $8 \mathrm{~m}$ mold and furnace. But the same paper also shows a 24 inch mirror that had been cast using very generally the same method we use today. In 1983 they cast two 72 inch mirrors using a mirror design and a mold design that would hardly change. And by 1983 they were committed to the right-side up, spinning design (option c in Figure 5) for $8 \mathrm{~m}$ mirrors. 


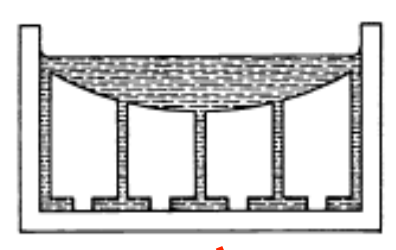

a.

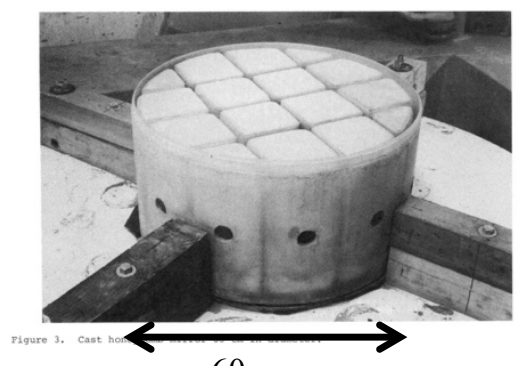

$60 \mathrm{~cm}$

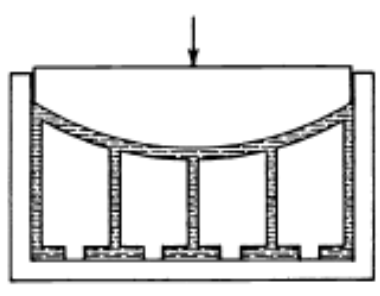

b.

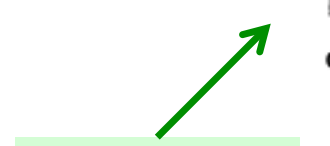

The eventual winner, by 1983

Figure 5. Multiple concepts for casting lightweight mirrors, from [11].

Looking ahead to $8 \mathrm{~m}$ telescopes, Angel saw short focal length as a huge advantage, in order to keep the mount short and stiff and the enclosure small and relatively inexpensive. He made the furnace spin to introduce the deep curve from the start. The next 72 inch mirror was cast in a spinning furnace whose $16 \mathrm{rpm}$ rotation gave an $\mathrm{f} / 1$ surface. ${ }^{[9]}$ When eventually scaled up to the $8.4 \mathrm{~m} \mathrm{f} / 1.14$ LBT primary mirrors, spin-casting would save 28 tons of expensive glass-more than the mass of the finished mirror - and would greatly reduce the annealing time and grinding time.

There was enough interest in these lightweight mirrors that Angel and Steward Observatory director Peter Strittmatter obtained support from the University of Arizona and the National Science Foundation to build the Mirror Lab along with a $12 \mathrm{~m}$ turntable, large enough to cast $8 \mathrm{~m}$ mirrors. Figure 6 shows the evolution of the turntable in 1984-86, from its foundation in what was then open space under the east stands of Arizona Stadium, to the initial furnace that was used to cast mirrors up to $3.5 \mathrm{~m}$ diameter. As the turntable was installed, walls and a ceiling were attached to the massive concrete stadium supports, to enclose the Mirror Lab.
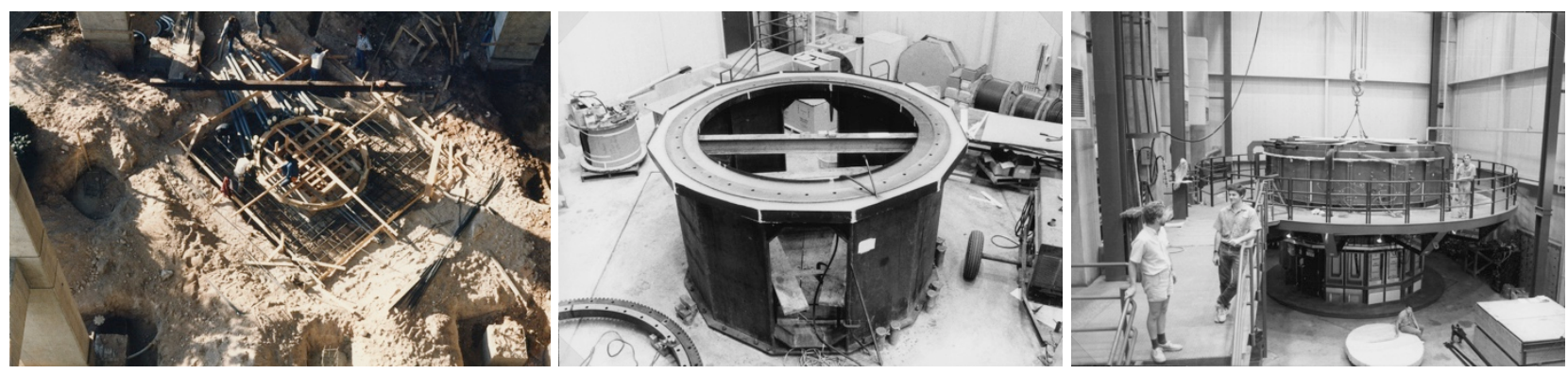

Figure 6. Left to right: turntable foundation under east stands of Arizona Stadium; turntable bearing installed in the (now enclosed) Mirror Lab; $12 \mathrm{~m}$ turntable with its initial furnace, used for mirrors up to $3.5 \mathrm{~m}$.

The first mirror cast was a puny 48 inch in 1987. Three $3.5 \mathrm{~m}$ mirrors followed in 1988-89, marking a breakthrough to metric units and providing primary mirrors for the Astrophysical Research Consortium's telescope at Apache Point Observatory, the Wisconsin-Indiana-Yale-NOAO telescope at Kitt Peak, and a US Air Force telescope at Starfire Optical Range. Simultaneously, we were developing a polishing system for fast mirrors, built around an active stressed lap 
whose shape was controlled to match the local curvature as it moved over the mirror. We polished the 72 inch $\mathrm{f} / 1$ mirror in an enclosed shop next to the turntable. ${ }^{[12]}$ This effort served to develop and demonstrate the possibility of making mirrors as fast as $\mathrm{f} / 1$, and resulted in a primary mirror for the Lennon Telescope of the Vatican Observatory, the first telescope at Mt. Graham International Observatory in Arizona.

The Starfire project provided much of the funding for a new polishing lab built in 1990 and equipped with an $8 \mathrm{~m}$ grinding and polishing machine and a $24 \mathrm{~m}$ test tower, which were first used to figure the $3.5 \mathrm{~m}$ mirrors. The Mirror Lab was not free to choose the focal ratios, so these mirrors were more conservative at $f / 1.5-f / 1.75$. During the same time frame we expanded the furnace to $8 \mathrm{~m}$ capacity, and in 1992-94 cast $6.5 \mathrm{~m}$ mirrors for the MMT (replacing the six 72 inch telescopes of the original Multiple Mirror Telescope) and the first of two Magellan telescopes at Las Campanas Observatory in Chile, future site of the GMT. As a partner in these telescope projects, Steward Observatory had a little more leverage in the choice of focal ratio, resulting in $\mathrm{f} / 1.25$.

The first $8.4 \mathrm{~m}$ mirror for LBT (f/1.14) was cast in 1997, followed by the second Magellan $6.5 \mathrm{~m}$ mirror in 1998 and the second LBT mirror in 2000. In 2003 the lab installed a second large polishing machine that's been used to polish all the $8.4 \mathrm{~m}$ mirrors starting with LBT2.

\section{TELESCOPES USING HONEYCOMB MIRRORS}

Figure 7 shows the LBT, at Mt. Graham International Observatory in Arizona. ${ }^{[13]}$ With two $8.4 \mathrm{~m}$ primary mirrors, it is the largest telescope on a single mount. Each half is a separate Gregorian telescope, and their wavefronts can be brought to focus on a common focal plane in a central beam combiner, giving the angular resolution of a $22 \mathrm{~m}$ telescope in one dimension. This coherent beam combination requires adaptive optics to deliver two well-corrected wavefronts into the beam combiner. Each half of LBT has a thin, actuated secondary mirror that serves as the deformable mirror for adaptive optics. Microgate and ADS (together now Adoptica) delivered the deformable mirror systems with 672 actuators each, using thin Zerodur shells $(910 \mathrm{~mm} \times 1.6 \mathrm{~mm})$ that were figured and thinned at the Mirror Lab. ${ }^{[14]}$
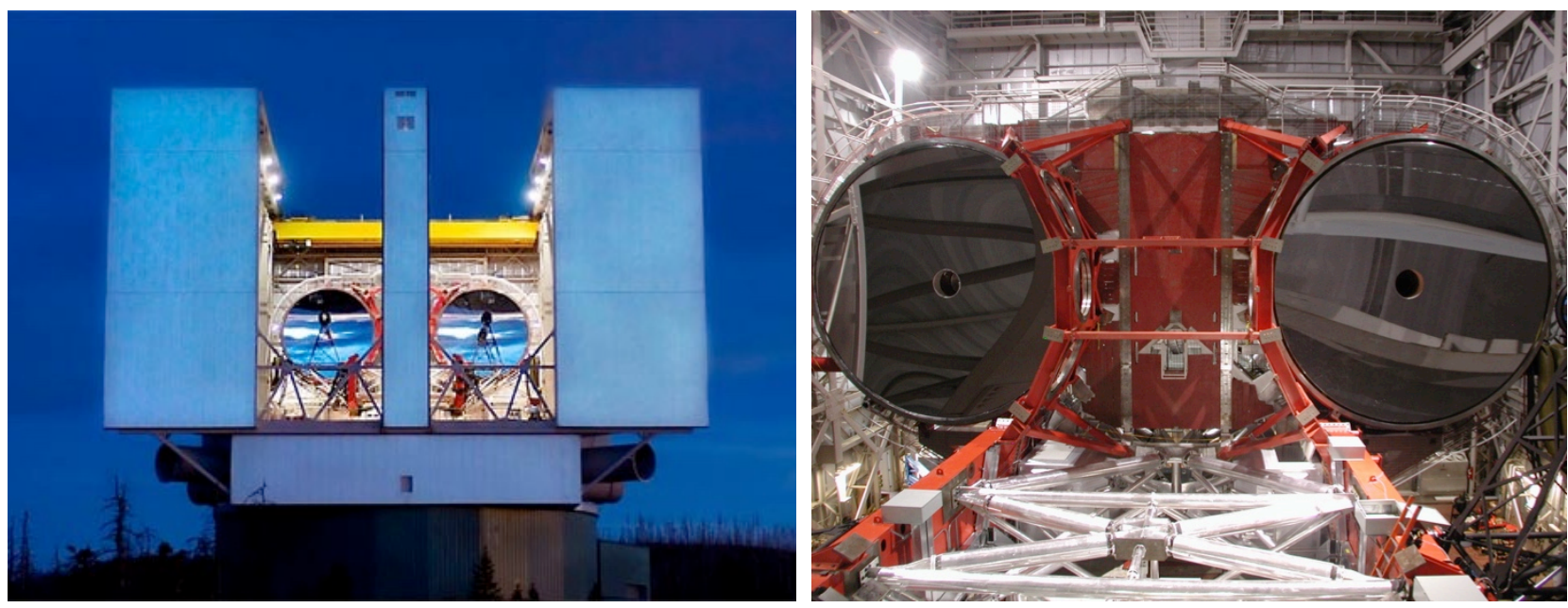

Figure 7. Large Binocular Telescope at Mt. Graham International Observatory in Arizona. LBT has the first two $8.4 \mathrm{~m}$ mirrors cast at the Mirror Lab. The common mount is shown at right.

The GMT, already shown in Figure 4, has a very different optical design with seven $8.4 \mathrm{~m}$ segments forming the primary mirror of a $25 \mathrm{~m} \mathrm{f} / 0.7$ Gregorian telescope. This design is dramatically different from the other two ELTS, whose primary mirrors are made of 500-800 $1.4 \mathrm{~m}$ segments. Using the largest segments that can be made guarantees a smooth wavefront over the largest possible subapertures, and minimizes the number of elements that must be controlled. In the GMT design, each $8.4 \mathrm{~m}$ primary segment is paired with a $1.1 \mathrm{~m}$ segment of the $3.2 \mathrm{~m}$ concave secondary mirror. Tight alignment requirements apply not to the individual segments but to the optical path through each pair of primary and secondary segments. This means the fine alignment and phasing will be performed with the small, agile secondary segments while the massive primary segments only need to be held in rough alignment to tens of microns, a relatively easy target. Like LBT, GMT's main adaptive optics system consists of deformable secondary mirror segments, each 
segment having about 700 actuators. These seven segments are ideal control elements for maintaining alignment and phasing for diffraction-limited performance.

GMT construction is underway on Las Campanas in Chile, where the Carnegie Observatories have operated telescopes for 50 years and near the site of the twin Magellan $6.5 \mathrm{~m}$ telescopes. Figure 8 shows the excavations for the telescope pier and enclosure.

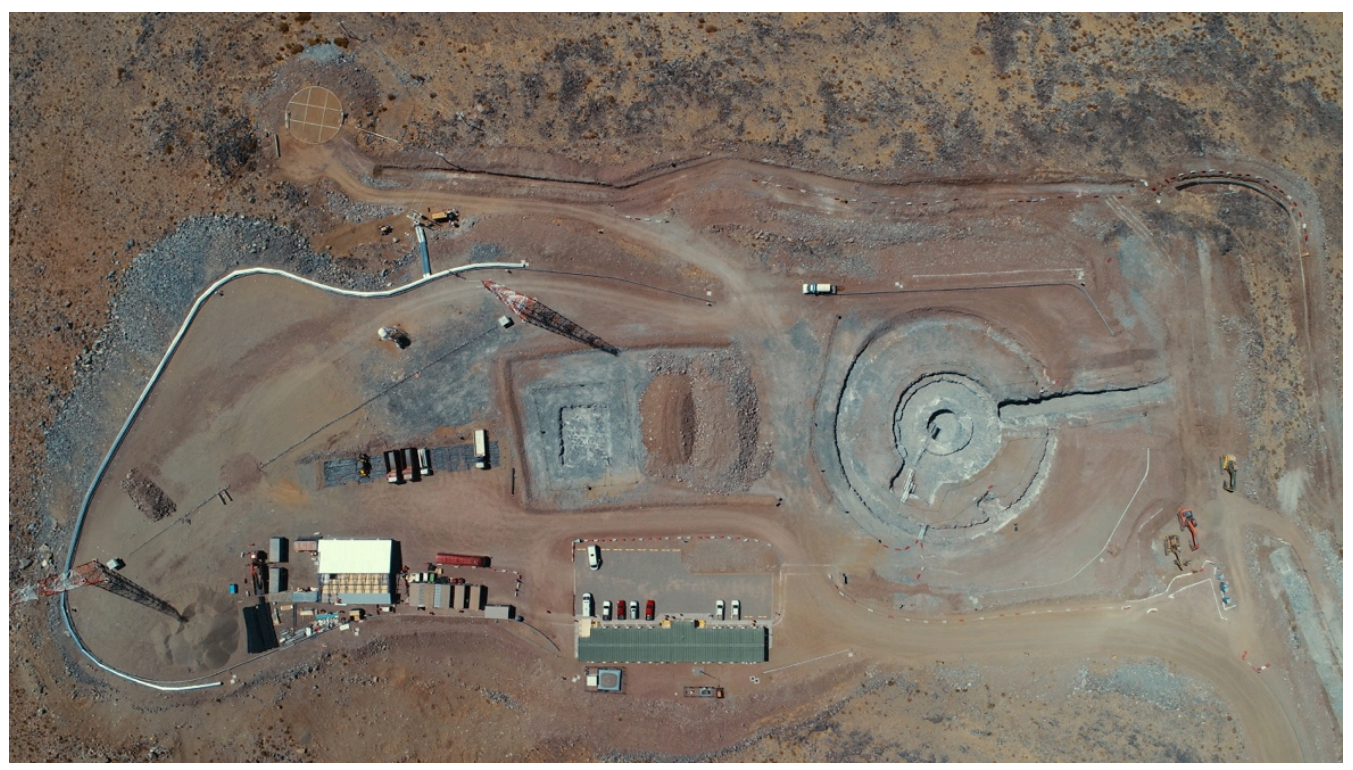

Figure 8. GMT site in March 2019, showing foundations for telescope pier and enclosure at right, and operations building at center. Courtesy of Giant Magellan Telescope - GMTO Corporation.

\section{MANUFACTURE OF THE GMT PRIMARY MIRROR SEGMENTS}

Discussion of a future ELT with seven $8.4 \mathrm{~m}$ segments began around 2001. The six outer segments would be off-axis sections of a $25 \mathrm{~m}$ near-parabolic parent. Off-axis mirrors presented new challenges in optical fabrication and testing, all related to the large and asymmetric aspheric departure of the optical surface. In the final GMT design, they would have $13 \mathrm{~mm}$ p-v departure, mostly astigmatism. The development included figuring an off-axis primary mirror for the New Solar Telescope at Big Bear Solar Observatory that was - through negotiation-nearly a $1 / 5$ scale model of a GMT segment. ${ }^{[15]}$

As the GMT project solidified, manufacture of the off-axis segments was seen as the most important technical challenge and therefore the highest priority for a technical demonstration. The first segment was cast in 2005. Over the next 7 years the Mirror Lab replaced the test tower (which had been built before GMT was conceived); made a $3.75 \mathrm{~m}$ spherical mirror that would go at the top of the new tower to fold the optical test and be part of the null corrector; developed an interferometric null test unlike anything ever built, as well as multiple independent measurements to confirm the accuracy on large and small scales; and carried out the optical fabrication of the segment. ${ }^{[16]}$ In parallel, we cast the $8.4 \mathrm{~m}$ combined primary and tertiary mirrors for the Large Synoptic Survey Telescope, a $6.5 \mathrm{~m}$ mirror for the Tokyo Atacama Observatory, and the second GMT segment. Since 2012 we have finished the second segment and cast three more, including the symmetric center segment. We are currently grinding and polishing these three segments.

Manufacture of primary mirrors has often been the bottleneck for telescope construction, as it was for the 100 inch telescope and the 200 inch telescope. We had serious challenges making the first GMT off-axis segment, but with two segments finished and three more moving through the production queue, the Mirror Lab is on schedule to deliver segments at a rate consistent with the project timeline. Figure 9 shows Segments 2-5 in recent states. Since these photos were taken, Segment 2 has been placed in its transport container and shipped to join Segment 1 in a storage facility in Tucson; and Segment 5 has been installed on the diamond generating machine where the rear surface is being prepared for attachment of its loadspreaders that interface with the mirror support system. 

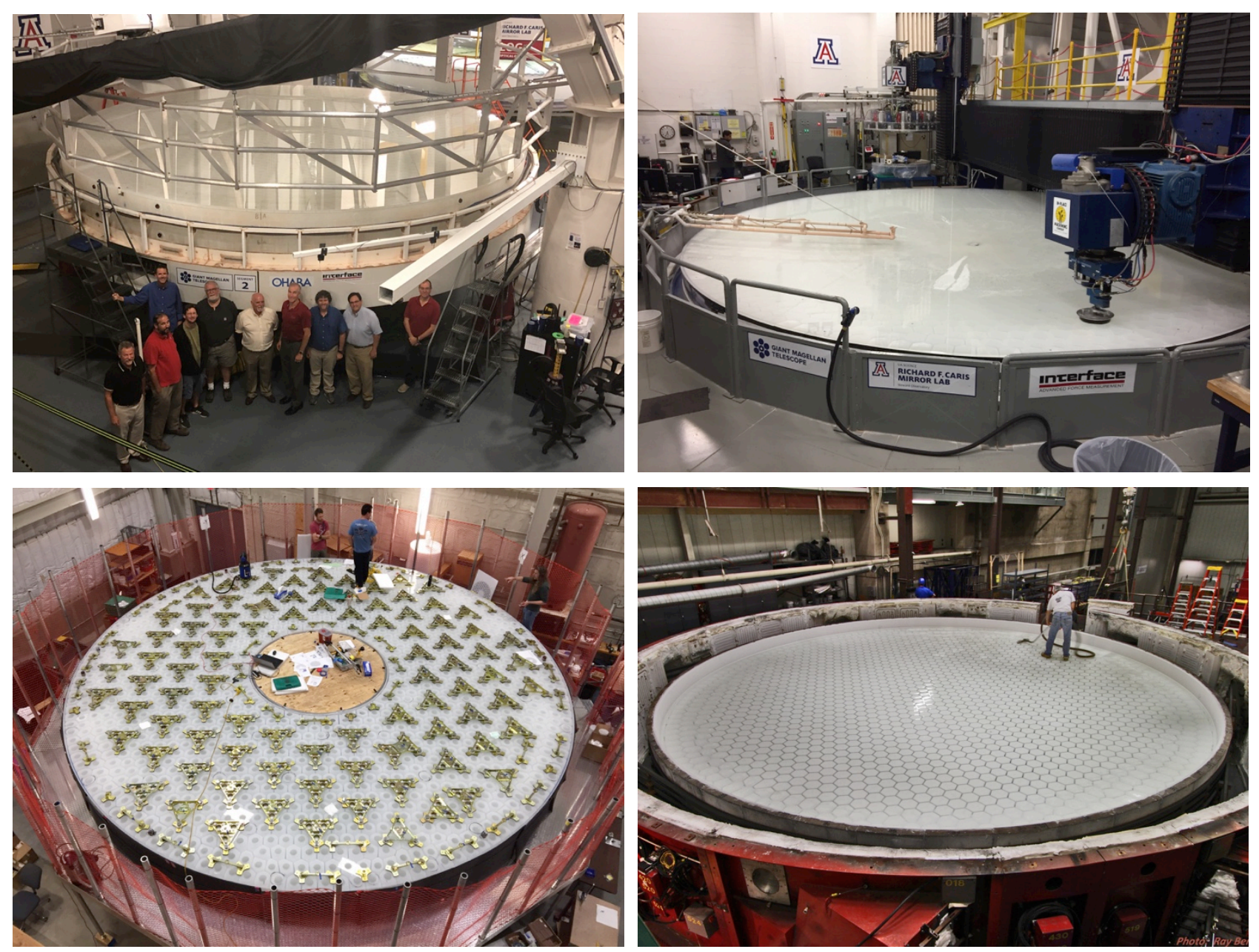

Figure 9. Top left: GMT Segment 2 at the time of the acceptance review in June 2019. Top right: Segment 3 undergoing fine grinding. Bottom left: Segment 4, the center segment, upside down after attachment of most of its loadspreaders. Bottom right: Segment 5, still in its mold and surrounded by the lower part of the furnace, at the completion of casting in February 2018. Photo of Segment 5 by Ray Bertram, Steward Observatory.

\subsection{Casting}

Spin-casting an $8.4 \mathrm{~m}$ honeycomb mirror is a dramatic event that's hard to describe adequately in words. The oral presentation of this paper includes a 6 minute video of the casting process. The file is too big to include with the paper, but the video can be seen on the Mirror Lab website, www.mirrorlab.arizona.edu. The process has been described in [17] and [18]. Almost all aspects are unchanged since the first $8.4 \mathrm{~m} \mathrm{LBT}$ mirror was cast in 1997. I will describe the process briefly here with photos, pointing out the few aspects that are unique to GMT segments.

The mold, shown in Figure 10, consists of a tub of silicon carbide cement, wrapped with Inconel steel bands to support its cylindrical wall, and lined with ceramic fiber. The cavities in the honeycomb mirror are formed by 1681 ceramic fiber boxes, most of them regular hexagons but including some irregular shapes near the edge in a transition to a ring of identical pentagons at the perimeter. Each box is bolted to the floor of the mold with silicon carbide hardware and a ceramic fiber spacer to hold the box $30 \mathrm{~mm}$ above the floor. The ceramic fiber (from Rex Materials, machined to final dimensions at the Mirror Lab) does not interact chemically with the glass, so it can be separated from the glass safely after the casting. The tops of the boxes follow the optical surface so the finished mirror will have a uniform facesheet, 28 $\mathrm{mm}$ thick. For an off-axis segment this means all 1681 boxes are unique, but the shapes have mirror symmetry across an axis that runs through the center of the parent surface. 


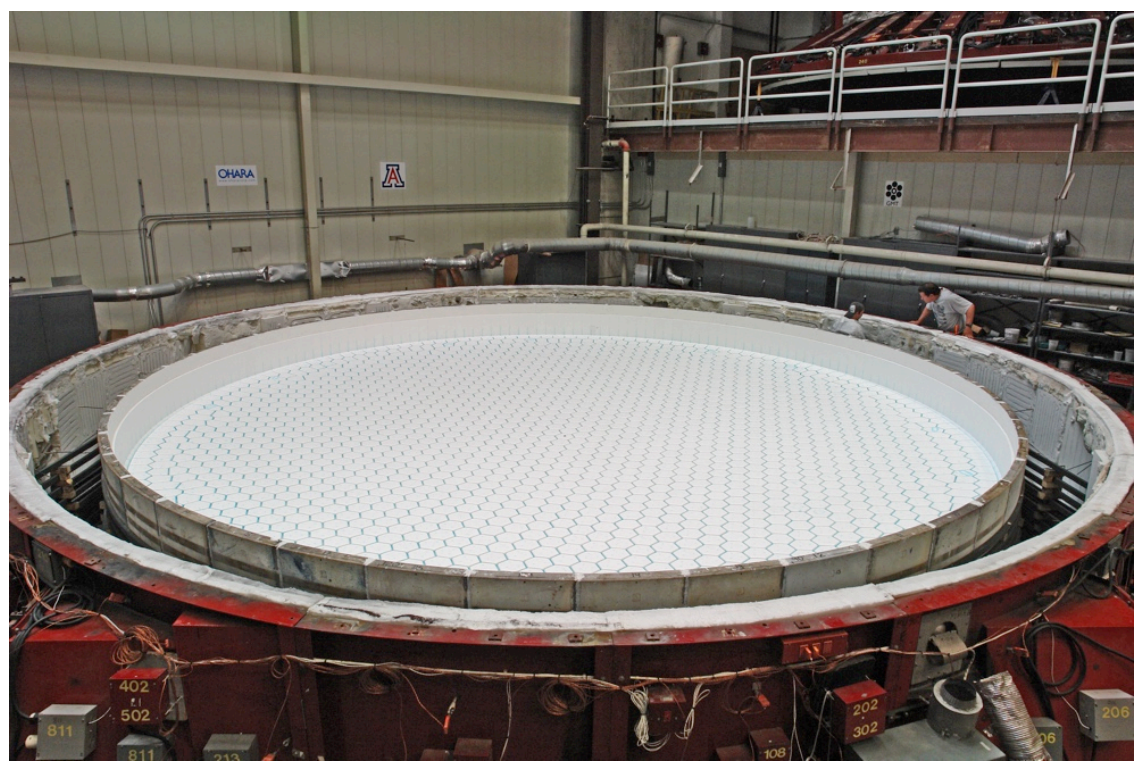

Figure 10. Mold for GMT Segment 2 (same for all off-axis segments) surrounded by the lower part of the furnace. The furnace lid can be seen at top right on its storage shelf. Photo by Ray Bertram, Steward Observatory.

The mold is pre-heated twice, once with the silicon carbide tub and steel bands, and once after installing the ceramic fiber pieces, in order to stabilize its dimensions. The glass - Ohara E6 low-expansion borosilicate - is then placed on top of the mold (Figure 11). For a GMT segment, $17,500 \mathrm{~kg}$ of glass is placed on the mold; grinding will reduce the mass of the finished mirror to about $16,000 \mathrm{~kg}$. The E6 glass is extremely uniform in expansion coefficient, which essentially eliminates distortion due to the change from lab temperature to operating temperature. A significant advantage for our process is that Ohara delivers the glass as blocks broken out of a set of one-ton melts. All surfaces are pristine fracture surfaces which melt together seamlessly in the honeycomb mold, leaving no trace of the original blocks.

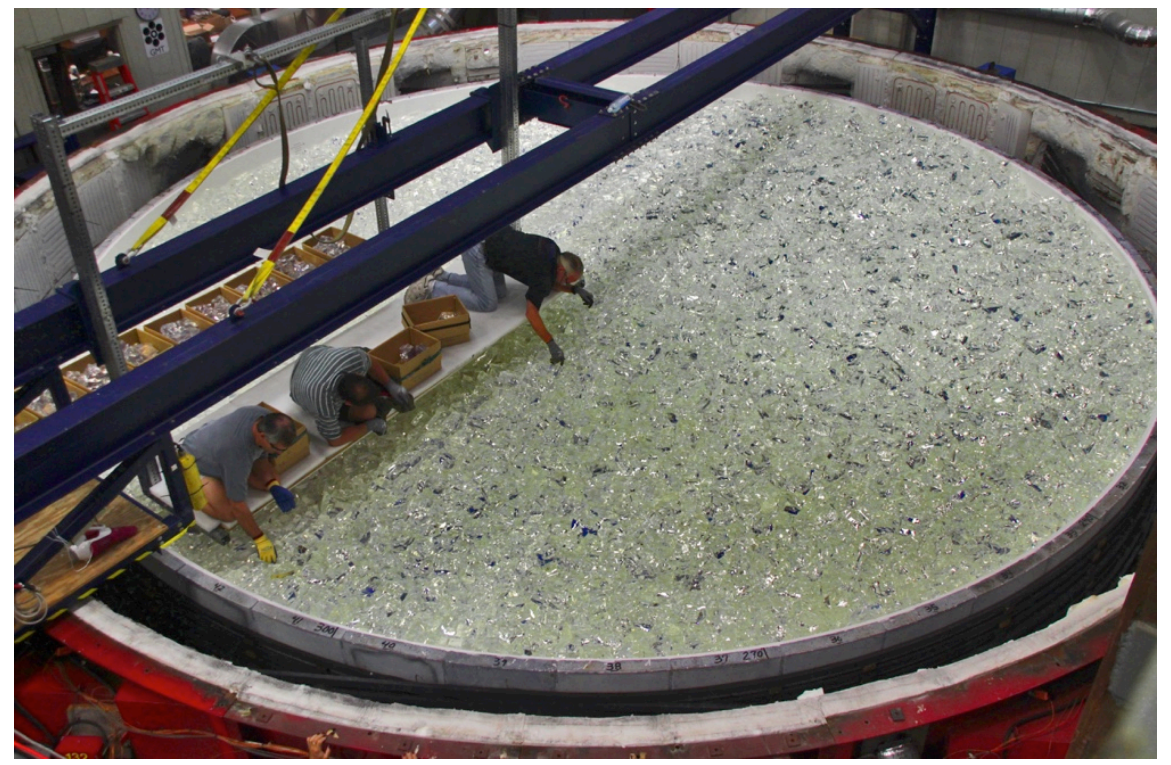

Figure 11. Placement of Ohara E6 glass blocks on the mold for GMT Segment 3. Photo by Ray Bertram, Steward Observatory.

The mold is enclosed in a furnace with electric coil heaters in the floor, walls and ceiling. It is heated over a few days to $1165^{\circ} \mathrm{C}$, where the glass has the consistency of cold honey. In a few hours the glass flows down the $12 \mathrm{~mm}$ gaps between 
boxes to form the ribs of the honeycomb mirror, and under the boxes to form the flat backplate. The furnace starts spinning before the glass starts to soften at $900^{\circ} \mathrm{C}$ and continues to spin until the glass is completely solid again. The rotation rate determines the sag of the glass surface. For a GMT segment it is $5 \mathrm{rpm}$ to give roughly the best-fitting paraboloid with $R=37 \mathrm{~m}$. (The off-axis optical surface, with its $13 \mathrm{~mm}$ p-v astigmatism, will be produced by diamond generating, which also removes several $\mathrm{mm}$ of excess glass from the front and rear surfaces.)

The mirror cools and anneals for 85 days. The cooling rate is only $3 \mathrm{~K} /$ day through the annealing range, $530-450^{\circ} \mathrm{C}$, in order to minimize the temperature gradients that will become density gradients with stress after the mirror reaches uniform temperature. Once the mirror cools to lab temperature, we remove the furnace in pieces and remove the mold's outer tub wall. At this point we can inspect the mirror's front facesheet and outer wall, but inspection of the internal structure has to wait until the floor of the mold and the ceramic fiber boxes are removed from inside the honeycomb.

To gain access to the back of the mirror for removal of the mold, we lift the mirror with a steel frame and turn it into a vertical plane. Most of the silicon carbide floor tiles and all of the ceramic fiber boxes are still attached, nearly doubling the weight supported. The lifting frame bonds to the mirror's front surface with compliant adhesive on 36 disks. The silicon carbide floor pieces and associated hardware are removed first using wrenches. This leaves a $90 \mathrm{~mm}$ hole in the backplate at the center of each honeycomb cell. We insert high-pressure water nozzles through these holes to break up the ceramic fiber and wash it out of the mirror. At this point we have a lightweight glass structure with the desired mechanical and thermal properties.

\subsection{Optical fabrication and testing}

Following the casting, we grind and polish the flat rear surface and attach the loadspreaders that form a permanent interface with the mirror support actuators. The mirror remains attached to the lifting frame for all work on the rear surface. The mirror is then turned right-side up and installed in a polishing cell which has the same support configuration as the operational (telescope) support system. The support forces are the same optimized zenith-pointing forces that will be used in the telescope. We diamond generate the optical surface to remove excess glass and match the off-axis prescription to an accuracy of $25 \mu \mathrm{m}$ rms.

We have used three types of tools for fine grinding and polishing GMT Segments 1 and 2: a $1.2 \mathrm{~m}$ stressed lap that bends actively to match the local curvature as it moves over the aspheric surface; compliant passive laps 10-40 cm in diameter containing a layer of non-Newtonian fluid (Silly Putty); and stiff passive pitch laps 5-40 cm in diameter. ${ }^{[19]} \mathrm{We}$ continue to explore different methods of using these tools and no doubt have room for improvement in methods and convergence rate, but we are making progress. Fine grinding and polishing of Segment 2 took less than half the calendar time as the same operations for Segment 1.

Development of tests for the GMT off-axis segments was a major challenge. Like all modern telescope mirrors, they are measured with an interferometer and a null corrector, shown in Figure 12. The null corrector converts a spherical wavefront into an accurate template wavefront for the off-axis mirror, including its $13 \mathrm{~mm}$ of asymmetric asphericity. It includes a tilted $3.75 \mathrm{~m}$ spherical mirror, a tilted $76 \mathrm{~cm}$ sphere, and a computer-generated hologram (CGH). The components are separated by up to $9 \mathrm{~m}$ and the distance from the large sphere to the GMT segment is $24 \mathrm{~m} .{ }^{[20]}$

The interferometric test is inherently accurate on scales up to a few meters on the GMT segment. The biggest concern is the accuracy of low-order aberrations (especially focus, astigmatism and coma), which are very sensitive to alignment errors in the test. Any aberrations present in the finished mirror can be corrected up to certain magnitudes by active optics at the telescope - a combination of repositioning the segment and bending it with the support actuators, based on feedback from a wavefront sensor. The accuracy requirement for manufacturing is that the errors are small enough to be corrected with active optics using a small, specified fraction of the range of adjustments. This translates to accuracies on the order of $1000 \mathrm{~nm}$ rms in focus ( $0.6 \mathrm{~mm}$ in radius of curvature), $300 \mathrm{~nm}$ rms in astigmatism, $100 \mathrm{~nm}$ rms in coma and trefoil, and diminishing levels of higher-order aberrations. These sound like loose requirements, but the corresponding alignment tolerances for the test are demanding. The larger dimensions must be controlled to about $100 \mu \mathrm{m}$, roughly the 3-dimensional accuracy of commercial laser trackers. The smaller dimensions such as alignment of the small sphere relative to the $\mathrm{CGH}$ must be controlled to about $10 \mu \mathrm{m}$. We developed a number of special alignment tools to achieve this accuracy. ${ }^{[21]}$ 


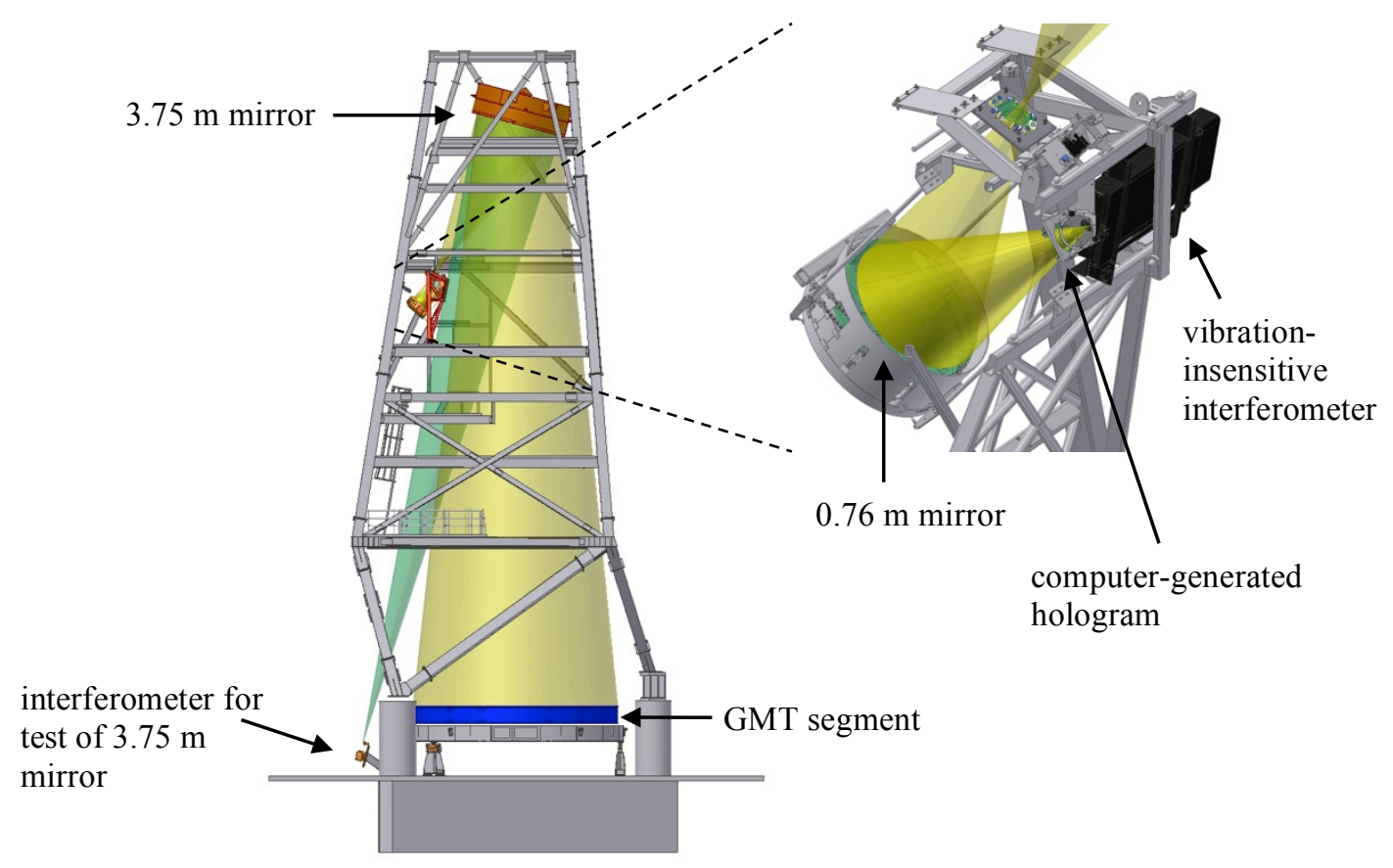

Figure 12. Model of the interferometric test for the GMT off-axis segments, in the $28 \mathrm{~m}$ test tower. At right is a blow-up of the interferometer and first two elements of the null corrector. Gold light cones represent the measurement of the GMT segment, while the aqua cone in the full model at left represents a simultaneous measurement of the $3.75 \mathrm{~m}$ fold sphere.

We can show on paper that the interferometric test achieves the required accuracy, but this doesn't guarantee against a mistake in assembly or measurement of the test configuration. We therefore measure the low-order aberrations independently with a scanning pentaprism test. ${ }^{[2],[23]}$ Unlike the interferometric test, it uses the primary mirror's natural geometry, the fact that it focuses collimated light to a point. We can't illuminate the full mirror segment with collimated light, but we can simulate it by scanning a small collimated beam across the mirror. This beam is parallel to the telescope's optical axis (tilted $13.5^{\circ}$ from vertical when the segment is face-up). We place a camera at the telescope's prime focus position, located by design at a corner of the test tower. In a perfect system the focused spot wouldn't move as the beam scans across the mirror (apart from a small, precisely known motion due to the fact that the mirror prescription is not exactly a paraboloid) but slope errors on the mirror cause it to move. Small motions of the scanning rail, GMT segment and camera also cause spot motion, so we add a fixed reference pentaprism at one end of the scanning rail, illuminating a position near the edge of the mirror. Differential motion between the scanning spot and the reference spot has uncontrolled variations of only $9 \mu \mathrm{m} \mathrm{rms}$, equivalent to $0.5 \mu \mathrm{rad} \mathrm{rms}$ wavefront slope, so it gives a very good measure of slope errors on the mirror. With scans across 5 different diameters, we can fit polynomials to the slope data to determine the first 8 low-order aberrations to accuracies similar to that of the interferometric measurement. Agreement between the two tests essentially guarantees that the mirror meets the requirements for large-scale shape.

A third independent test, a 2-D scan of the surface with a laser tracker, measures focus to about the same accuracy as the pentaprism test (both slightly better than the interferometric test) and helps reduce the uncertainty in the weighted average or best estimate of radius of curvature. ${ }^{[24],[25]}$ This is especially important with seven segments that must match in radius. The matching requirement is agreement within the range of correction with active optics, i. e. perfect matching after the correction.

Figure 13 shows the results of the fabrication process for GMT Segments 1 and 2. The plots show the surface error after we simulate the active optics correction that will be made at the telescope. Both segments meet the accuracy requirements, and it's not surprising that the second segment is more accurate at $14 \mathrm{~nm} \mathrm{rms}$ than the first (22 $\mathrm{nm} \mathrm{rms})$. More significant than the improved accuracy is the fact that the fine grinding and polishing operations took less than half the time for Segment 2. 

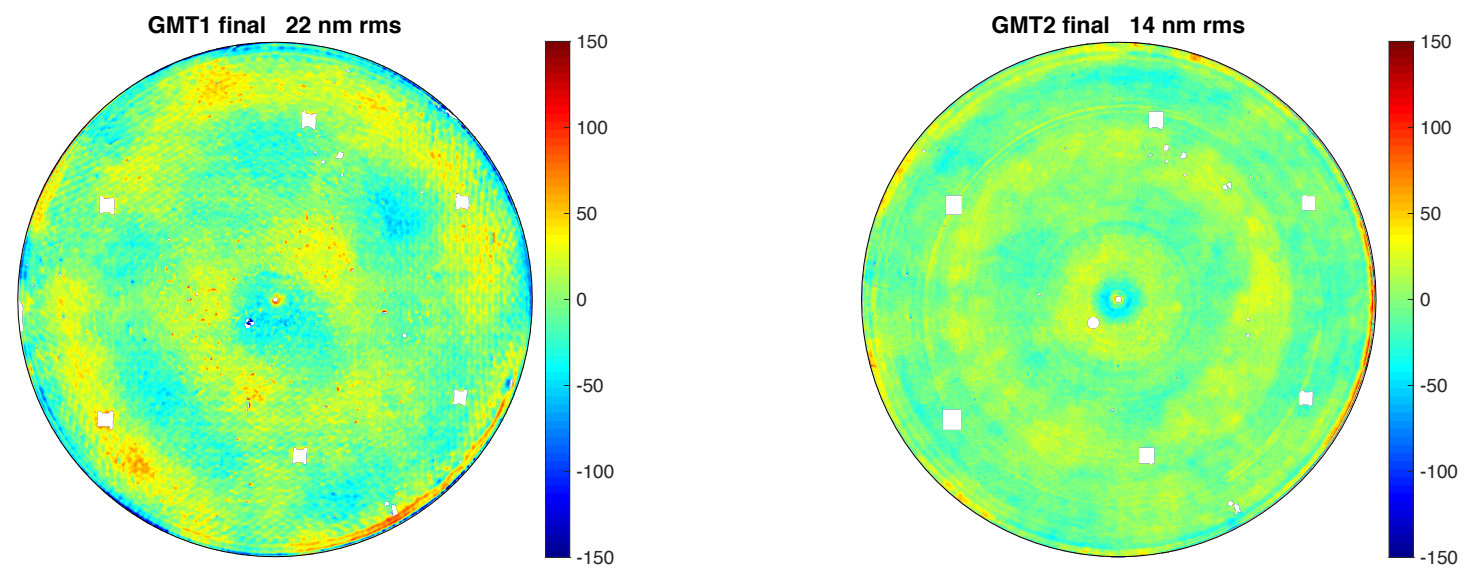

Figure 13. Finished surfaces of GMT Segments 1 and 2. Plots show surface error in nm, after simulated active optics correction using segment alignment and 27 bending modes. These corrections use a small fraction of the available motion and actuator force range.

\section{SCIENCE WITH ELTS}

The range of astrophysics to which GMT will contribute is presented in [26] and goes far beyond the scope of this paper. I want to highlight one area that will benefit tremendously from the sensitivity and angular resolution of GMT and the other ELTs. That is the study of exoplanets, planets around stars other than our Sun. Thousands of exoplanets have been detected indirectly, either by the tiny orbital motion they induce in their parent stars or (for planets whose orbital planes nearly intersect Earth) by the reduction in their star's brightness when they pass between the star and Earth. Figure 14 shows the periodic radial velocity of our nearest-neighbor star, Proxima Centauri. The star moves at $5 \mathrm{~km} / \mathrm{hr}$ - walking speed - with an 11-day period. The planet causing this motion probably has a temperature that could allow liquid water, but that's because it's orbiting very close to a small star.

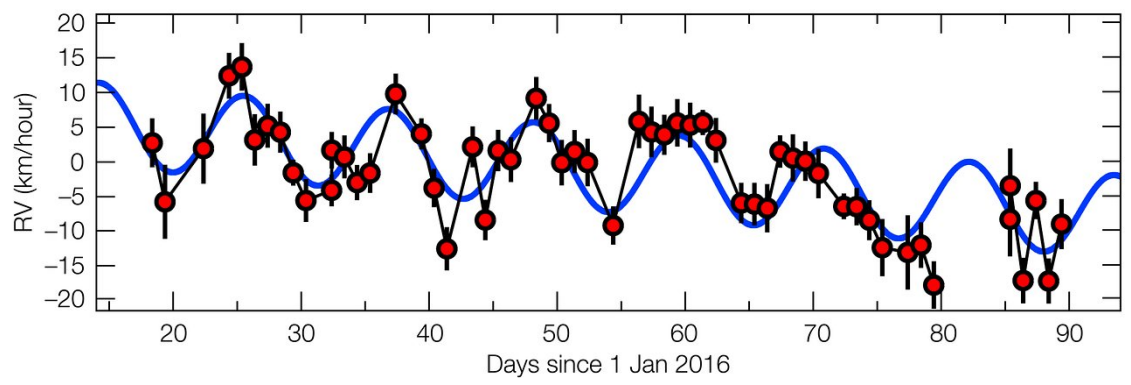

Figure 14. Reflex velocity of the star Proxima Centauri due to a planet orbiting with an 11-day period. Data are from [27].

Our Sun's reflex velocity due to Earth is $0.32 \mathrm{~km} / \mathrm{hr}$, crawling speed! Detecting a true Earth-like planet in this way requires a spectrograph that's more sensitive and more stable than the current generation, and this level is the goal for the next generation of spectrographs including the GMT-Consortium Large Earth Finder (G-CLEF). As the name suggests, the expectation is to find planets a bit larger than Earth, and even this would be a great technical achievement. Still, the great potential impact of the ELTs is not more indirect detections, but direct imaging and spectroscopy of planets. For this, the huge challenge is separating the planet from its star which is on the order of a billion times brighter and-for an earth-like planet orbiting one of the 1000 nearest stars - only half a micro-radian away from the planet. For direct detection of Earth-like planets, we need GMT's diffraction-limited resolution of $40 \mathrm{nrad}$ at $\lambda=1 \mu \mathrm{m}$, exquisite wavefront control from future adaptive optics such as the GMagAO-X system being developed for GMT, and an advanced coronograph to block the light from the star. 
And the holy grail of exoplanet science is not just a point-like image of a planet; it's a spectrum of reflected light from the planet-like the model in Figure 15-containing a signature of life in the form of absorption lines from the planet's atmosphere, including oxygen and a combination of other molecules that couldn't persist without continuous input from living organisms. For this, the sensitivity of a $25 \mathrm{~m}$ or larger telescope greatly extends the range and increases the number of candidates for discovery of life.

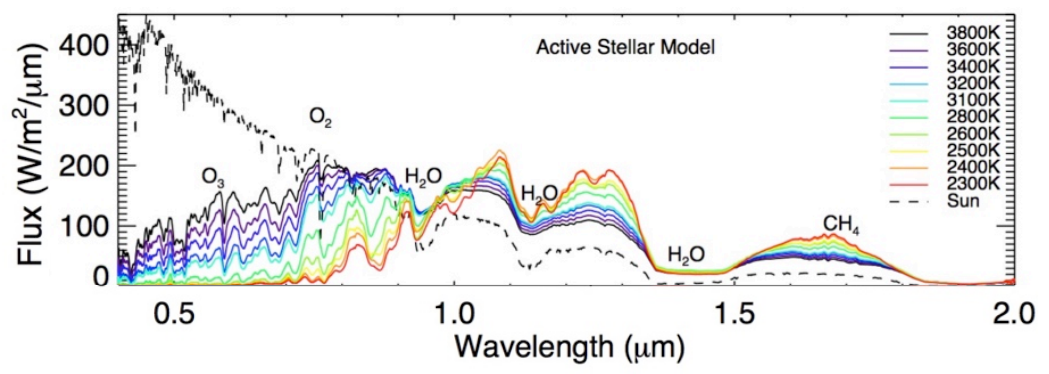

Figure 15. Model spectrum of an exoplanet in reflected light, showing absorption lines from oxygen, ozone, water and methane, from [28].

Finding life will require not only giant telescopes but continued evolution of the instruments that make them so valuable. It's not going to happen at first light with any ELT. It might not happen in my lifetime, but unless life requires an extraordinary convergence of extremely rare conditions, it will happen during the lives of young people who are starting to learn about the Universe today.

And who knows whether finding life will be the next big discovery, in the same category as Hubble's discovery of the expanding Universe with the 100 inch telescope nearly 100 years ago. It could be something completely unexpected. There's no telling what we'll learn when we double the size of the telescope.

\section{ACKNOWLEDGMENTS}

This paper represents the work of a lot of people at (or formerly at) Steward Observatory and the College of Optical Sciences, including Rich Allen, Roger Angel, Jim Burge, Roger Ceragioli, Warren Davison, John Hill, Buell Jannuzi, Dae Wook Kim, Jeff Kingsley, Cary Kittrell, Kevin Law, Adrian Loeff, Andrew Lowman, Randy Lutz, Tom McMahon, Chang Jin Oh, Greg Smith, Peter Strittmatter, Peng Su, Mike Tuell, Stuart Weinberger, Steve West, Chunyu Zhao, Ping Zhou and Tom Zobrist.

This work has been supported by the GMTO Corporation, a non-profit organization operated on behalf of an international consortium of universities and institutions: Arizona State University, Astronomy Australia Ltd, the Australian National University, the Carnegie Institution for Science, Harvard University, the Korea Astronomy and Space Science Institute, the São Paulo Research Foundation, the Smithsonian Institution, the University of Texas at Austin, Texas A\&M University, the University of Arizona, and the University of Chicago.

\section{REFERENCES}

[1] S. Weinberg, Gravitation and Cosmology: Principles and Applications of the General Theory of Relativity, John Wiley \& Sons, Inc., New York, pp. 417, 445 (1972).

[2] R. N. Wilson, Reflecting Telescope Optics I, Springer-Verlag, Berlin, ch. 5 (1996).

[3] D. E. Osterbrock, Pauper and Prince: Ritchey, Hale and Big American Telescopes, The University of Arizona Press, Tucson \& London (1993).

[4] J. E. Nelson, T. S. Mast, and S. M. Faber, "The design of the Keck Observatory and Telescope," Keck Observatory Report 90 (W. M. Keck Observatory, Kamuela, Hawaii, 1985).

[5] F. Liu and G. Sanders, "Thirty Meter telescope project status (Conference Presentation)," Proc. SPIE 10700, Ground-based and Airborne Telescopes VII, 1070013 (10 July 2018).

[6] R. Tamai, B. Koehler, M. Cirasuolo, F. Biancat-Marchet, M. Tuti and J. C. Gonzáles Herrera, "The ESO's ELT construction status," Proc. SPIE 10700, Ground-based and Airborne Telescopes VII, 1070014 (6 July 2018). 
[7] R. N. Wilson, F. Franza and L. Noethe, "Active optics I. A system for optimizing the optical quality and reducing the costs of large telescopes," JMO 34, 485-509 (1987).

[8] R. N. Wilson, F. Franza, L. Noethe and G. Andreoni, "Active optics IV. Set-up and performance of the optics of the ESO New Technology Telescope (NTT) in the observatory," JMO 38, 219-243 (1991).

[9] L. Goble, J. R. P. Angel and J. M. Hill, "Steps toward $8 \mathrm{~m}$ honeycomb mirrors VII. Spin casting an experimental f/1 $1.8 \mathrm{~m}$ honeycomb blank of borosilicate glass," Proc. SPIE 571, Large Optics Technology (1985).

[10] J. Fanson, P. J. McCarthy, R. Bernstein, G. Angeli, D. Ashby, B. Bigelow, A. Bouchez, W. Burgett, E. Chauvin, A. Contos, F. Figueroa, P. Gray, F. Groark, R. Laskin, R. Millan-Gabet, A. Rakich, R. Sandoval, M. Pi and N. Wheeler, "Overview and status of the Giant Magellan Telescope project," Proc. SPIE 10700, Ground-based and Airborne Telescopes VII, 1070012 (6 July 2018).

[11] J. R. P. Angel and J. M. Hill, "Manufacture of large glass honeycomb mirrors," Proc. SPIE 332, Advanced Technology Optical Telescopes (1982).

[12] H. M. Martin, D. S. Anderson, J. R. P. Angel, J. H. Burge, W. B. Davison, S. T. DeRigne, B. B. Hille, D. A. Ketelsen, W. C. Kittrell, R. McMillan, R. H. Nagel, T. J. Trebisky, S. C. West and R. S. Young, "Stressed-lap Polishing of 1.8-m f/1 and 3.5-m f/1.5 Primary Mirrors", Conference on Progress in Telescope and Instrumentation Technologies, ed. M.-H. Ulrich, ESO Conference and Workshop Proc. 42, p. 169 (1992).

[13] J. M. Hill, R. F. Green, D. S. Ashby, J. G. Brynnel, N. J. Cushing, J. K. Little, J. H. Slagle, R. M. Wagner, "The Large Binocular Telescope," Proc. SPIE 8444, Ground-based and Airborne Telescopes IV, 84441A (17 September 2012).

[14] A. Riccardi, M. Xompero, D. Zanotti, L. Busoni, C. Del Vecchio, P. Salinari, P. Ranfagni, G. Brusa Zappellini, R. Biasi, M. Andrighettoni, D. Gallieni, E. Anaclerio, H. M. Martin and S. M. Miller, "Adaptive secondary mirror for the Large Binocular Telescope: results of acceptance laboratory test”, Adaptive Optics Systems, Proc. SPIE 7015, $701512(2008)$.

[15] H. M. Martin, J. H. Burge, S. M. Miller, B. K. Smith, R. Zehnder and C. Zhao, "Manufacture of a 1.7 m prototype of the GMT primary mirror segments", Optomechanical Technologies for Astronomy, Proc. SPIE 6273 (2006).

${ }^{[16] ~ H . ~ M . ~ M a r t i n, ~ R . ~ G . ~ A l l e n, ~ J . ~ H . ~ B u r g e, ~ D . ~ W . ~ K i m, ~ J . ~ S . ~ K i n g s l e y, ~ K . ~ L a w, ~ R . ~ D . ~ L u t z, ~ P . ~ A . ~ S t r i t t m a t t e r, ~ P . ~ S u, ~ M . ~}$ T. Tuell, S. C. West and P. Zhou, "Production of $8.4 \mathrm{~m}$ segments for the Giant Magellan Telescope", Proc. SPIE 8450, Modern Technologies in Space- and Ground-based Telescopes and Instrumentation II, 84502D (2012).

[17] B. H. Olbert, J. R. P. Angel, J. M. Hill and S. F. Hinman, "Casting 6.5-meter mirrors for the MMT conversion and Magellan," Proc. SPIE 2199, Advanced Technology Optical Telescopes V, (1 June 1994).

[18] J. M. Hill, J. R. P. Angel, R. D. Lutz, B. H. Olbert and P. A. Strittmatter, "Casting the first 8.4-m borosilicate honeycomb mirror for the Large Binocular Telescope," Proc. SPIE 3352, Advanced Technology Optical/IR Telescopes VI, (25 August 1998).

[19] D. W. Kim, J. H. Burge, J. M. Davis, H. M. Martin, M. T. Tuell, L. R. Graves and S. C. West, "New and improved technology for manufacture of GMT primary mirror segments," Proc. SPIE 9912, Advances in Optical and Mechanical Technologies for Telescopes and Instrumentation II, 99120P (22 July 2016).

[20] J. H. Burge, W. Davison, H. M. Martin and C. Zhao, "Development of surface metrology for the Giant Magellan Telescope primary mirror", Proc. SPIE 7018, Advanced Optical and Mechanical Technologies in Telescopes and Instrumentation, 701814 (2008).

[21] S. C. West, J. H. Burge, B. Cuerden, W. B. Davison, J. Hagen, H. M. Martin, M. T. Tuell and C. Zhao, "Alignment and use of the optical test for the $8.4 \mathrm{~m}$ off-axis primary mirrors of the Giant Magellan Telescope", Proc. SPIE 7739, Modern Technologies in Space- and Ground-based Telescopes and Instrumentation, 77390N (2010).

[22] P. Su, J. H. Burge, B. Cuerden and H. M. Martin, "Scanning pentaprism measurements of off-axis aspherics", Proc. SPIE 7018, Advanced Optical and Mechanical Technologies in Telescopes and Instrumentation, 70183T (2008).

[23] P. Su, J. H. Burge, B. Cuerden, R. G. Allen and H. M. Martin, "Scanning pentaprism measurements of off-axis aspherics II," Proc. SPIE 7426, Optical Manufacturing and Testing VIII, 74260Y (2009).

[24] T. Zobrist, J. H. Burge, W. Davison and H. M. Martin, "Measurement of large optical surfaces with a laser tracker", Proc. SPIE 7018, Advanced Optical and Mechanical Technologies in Telescopes and Instrumentation, 70183U (2008).

[25] T. L. Zobrist, J. H. Burge and H. M. Martin, "Accuracy of laser tracker measurements of the GMT 8.4 m off-axis mirror segments", Proc. SPIE 7739, Modern Technologies in Space- and Ground-based Telescopes and Instrumentation, 77390S (2010).

[26] GMT Science Book 2018, GMTO Corporation. 
[27] G. Anglada-Escudé, et al., "A terrestrial planet candidate in a temperate orbit around Proxima Centauri," Nature 536, 437-440 (25 August 2016).

[28] S. Rugheimer, et al., "Effect of UV radiation on the spectral fingerprints of Earth-like planets orbiting M stars," ApJ 809, 57-72 (2015). 